Brief note

\title{
NATURAL FREQUENCIES OF AXISYMMETRIC VIBRATIONS OF THIN HYPERBOLIC CIRCULAR PLATES WITH CLAMPED EDGES
}

\author{
J. JAROSZEWICZ \\ Faculty of Management \\ Bialystok University of Technology \\ 2 Ojca Stefana Tarasiuka St., 16-001 Kleosin, POLAND \\ E-mail: j.jaroszewicz@pb.edu.pl
}

\begin{abstract}
A free vibration analysis of homogeneous and isotropic circular thin plates with nonlinear thickness variation and clamped edges is considered. The limited independent solutions of differential Euler equation were expanded in the power series based on the properties of integral equations. The analytical frequency equations as power series were obtained using the method of successive approximations.
\end{abstract}

Key words: circular plates, power series, clamped edge.

\section{Introduction}

The study of the vibration of a thin circular plate is basic in structural mechanics. Components of circular plates are commonly used in aerospace industries and aviation as well as in marine and civil engineering applications. The natural frequencies of circular plates have been studied extensively for more than a century. If only the frequency of the external load matches the natural frequency of the plate, destruction may occur. Knowledge about the distribution of variable values of mass and stiffness can allow shaping the dynamic behavior of structural elements such us circular and annular plates.

The free vibration of circular plates of constant and variable thickness has received considerable attention in the literature. The work of Leissa [1] is an excellent source of information about methods used for free vibration analysis of plates. Conway [2,3] analyzed the axisymmetric vibration of thin circular plates with a power function thickness variation under particular Poisson's ratio in terms of Bessel functions. Jain et al. [4] studied the axisymmetric vibration of thin circular plates of linearly varying thickness using by Frobenius method. Wang [5] used the power series method for free vibration analysis of circular thin plates of power varying thickness. Wu and Liu [6,7] proposed a generalized differential quadrature rule (GDQR) for the free vibration analysis of circular thin plates of constant and variable thickness. Jaroszewicz and Zoryj [8] studied free vibration of circular thin plates of variable distribution of parameters using the method of partial discretization (MPD). Zhou et al. [9] applied a Hamiltonian approach to the solution of a free vibration problem of circular and annular thin plates. Duan et al. [10] proposed the DSC element method for the free vibration analysis of circular thin plates with constant and stepped thickness. Żur [11, 12] analyzed free vibration of thin circular and annular plates of constant and nonlinear variable thickness.

This article examines the circular plate whose thickness decreases with the distance from the centre of symmetry. Any profile with a sufficient approximation can be replaced with a hyperbole with the index $m<0$. Natural frequencies of axisymmetric vibrations of a circular plate with clamped edges were determined on the basis of the solution of an appropriate boundary problem.

The paper presents a hyperbolic circular plate made of different materials, e.g., aluminum and tin. It has been shown that the effect of Poisson's ratio on the coefficient of the fundamental frequency in the case $m<0$ (disc) is different than in the case $m>0$, because if it increases from 0 to 0.5 , the factor is monotonically 
increasing in the case of the disc. The method of influence function, characteristic series and Bernstein estimates are used. The studied case corresponds to $m=-1$ and values of Poisson's ratio in the range $0<v$ $<0.5$. By way of example, the effect of material and resilient constants have been examined for discs made of aluminum and tin, and a conclusion was drawn for the effect of the parameter $\mathrm{m}(m=-1, m=2)$ on the basic frequency, which was confronted with the case of the plate with constant thickness $(m=0)$.

\section{Statement of the problem}

The subject of the examination was a clamped, circular plate of the radius $R$ (Fig.1) whose stiffness $D$ and thickness $h$ are power functions of the radial coordinate $r$

$$
\begin{aligned}
& D=D_{0}\left(\frac{r}{R}\right)^{m} ; \quad h=h_{0}\left(\frac{r}{R}\right)^{\frac{m}{3}} ; \quad m \leq 0, \\
& 0<r \leq R ; \quad D_{0}=\frac{E h_{0}^{3}}{12\left(1-v^{2}\right)}
\end{aligned}
$$

where: $D_{0}, h_{0}$ and $m$ are set constants.

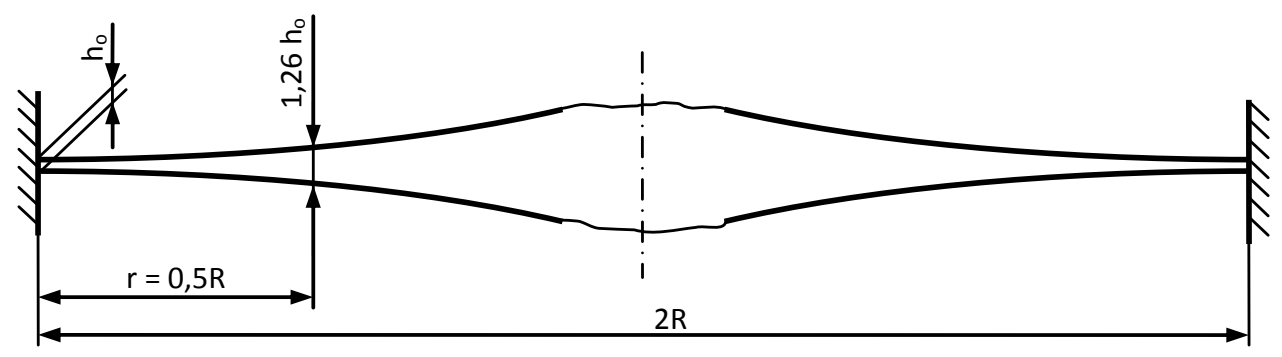

Fig.1. A model of examined plate, Jaroszewicz and Zoryj [14].

The analysis of natural, transverse and axisymmetric vibrations of such plates is carried out on the basis of an adequate boundary problem

$$
\begin{aligned}
& L_{0}[u]-p r^{-\frac{2}{3} m} u=0 ; \quad p=\frac{\rho h_{0}}{D_{0}} R^{\frac{2}{3} m} \omega^{2}, \\
& u(R)=0 ; \quad u^{\prime}(R)=0, \\
& L_{0}[u] \equiv u^{I V}+\frac{2}{r}(m+1) u^{I I I}+\frac{1}{r^{2}}\left(m^{2}+m+v m-1\right) u^{I I}+\frac{1}{r^{3}}(m-1)(v m-1) u^{I}
\end{aligned}
$$

where: $u=u(r)$ - the amplitude of deflection, $\rho$ - the specific density of a material, $\omega$ - the frequency, $v$ - Poisson's coefficient, E - Young's modulus. The following case shall be examined, where $m=-1$. Then 


$$
L_{0}[u] \equiv u^{I V}-\frac{1+v}{r^{2}} u^{I I}+\frac{2(1+v)}{r} u^{I}
$$

where Eqs (2.2) takes the character below

$$
L_{0}[u]-p r^{\frac{2}{3}} u=0 ; \quad p=\frac{\rho h_{0}}{D_{0}} R^{-\frac{2}{3}} \omega^{2}
$$

First of all, linear independent solutions of the following equation are found $L_{0}[u]=0$

$$
\begin{aligned}
& 1 ; \quad r^{3} ; \quad r_{1}^{\mu} ; \quad r_{2}^{\mu} ; \\
& \mu_{1}=\frac{1}{2}(3+\sqrt{5+4 v}) ; \quad \mu_{2}=\frac{1}{2}(3-\sqrt{5+4 v}) .
\end{aligned}
$$

It is worth noting that only the first two solutions, along with their derivatives of any order, are limited, and the next two have unlimited derivatives, starting from the derivative of the third row, since $0<v<0.5$. Hence the solutions of Eq.(2.6) can be written in the following form

$$
\begin{aligned}
& U=1+p u_{1}+p^{2} u_{2}+\ldots ; \quad V=r^{3}+p \mathrm{v}_{1}+p^{2} \mathrm{v}_{2}+\ldots ; \\
& u_{i}=\int_{0}^{r} K_{0}(r, s) s^{\frac{2}{3}} u_{i-1}(s) d s ; \quad u_{0}=1 ; \\
& \mathrm{v}_{i}=\int_{0}^{r} K_{0}(r, s) s^{\frac{2}{3}} v_{i-1}(s) d s ; \quad \mathrm{v}_{0}=r^{3} ; \quad(i=1,2, \ldots) ; \\
& K_{0}(r, \alpha)=\frac{1}{2 \sqrt{5+4 v}}\left(r^{\mu_{2}} \alpha^{\mu_{1}}-r^{\mu_{1}} \alpha^{\mu_{21}}\right)+\frac{1}{6}\left(r^{3}-\alpha^{3}\right)
\end{aligned}
$$

where $K_{0}(r, \alpha)$ - Cauchy's function, multiplied by Heaviside's function is a function of the impact or the fundamental function of the operator $L_{0}[u]$. When the integrals are designated (2.10) we can find solutions (2.9) which are quick-running series. In particular, when it is limited to $i=1,2$, the result is Jaroszewicz and Zoryj [14]

$$
\begin{aligned}
& U=1+p a(v) r^{\frac{14}{3}}+p^{2} a(v) b(v) r^{\frac{28}{3}} ; \quad V=r^{3}+p c(v) r^{\frac{23}{3}}+p^{2} c(v) d(v) r^{\frac{37}{3}} ; \\
& a(v)=\frac{81(1-v)}{140(79-9 v)} ; \quad b(v)=\frac{81(1-v)}{28 \cdot 38(541-9 v)} ; \\
& c(v)=\frac{81(1-v)}{28 \cdot 38(331-9 v)} ; \quad d(v)=\frac{81(1-v)}{28 \cdot 74(1045-9 v)} .
\end{aligned}
$$


Thus, the limited (for $r=0$ ) solution of Eq.(2.6) will be defined by the formula $u(r)=A U+B V$, where $\mathrm{A}$ and $\mathrm{B}$ are arbitrary integral constants. By substituting them to the boundary conditions (2.3), the equation of frequency is obtained

$$
\left|\begin{array}{cc}
U & V \\
U^{\prime} & V^{\prime}
\end{array}\right|_{r=R}=0
$$

\section{Solution of the problem}

Acting on Eq.(2.14) and taking Eqs (2.6), (2.12) and (2.13) into consideration, it was possible to determine the first three coefficients of a distinctive series in the form of a power series with respect to the frequency parameter

$$
\begin{aligned}
& A_{0}-A_{1} \Lambda+A_{2} \Lambda^{2}-\ldots=0 ; \\
& A_{0}=3 ; \quad A_{1}=\frac{1}{3}[23 c(v)-5 a(v)] \\
& A_{2}=\frac{1}{3}[-19 a(v) b(v)+9 a(v) c(v)+37 c(v) d(v)]
\end{aligned}
$$

where

$$
\Lambda=\frac{\rho h_{0}}{D_{0}} \omega^{2} R^{4}
$$

Referring to formulas (2.13) - (3.2), $A_{l}(v)$ and $A_{2}(v)$ can be calculated for selected frequencies $v$, where $A_{0} \equiv 1$. The results of these calculations are presented in Tab.1.

Table 1. Values of the first two coefficients of the distinctive series.

\begin{tabular}{|c|c|c|c|c|c|c|}
\hline$v$ & 0 & $1 / 9$ & $2 / 9$ & $3 / 9$ & $4 / 9$ & 0,5 \\
\hline$A_{1}(v)$ & 0.0092928 & 0.0083915 & 0.007461 & 0.0064991 & 0.0055045 & 0.0049951 \\
\hline$A_{2}(v)$ & 0.000002 & 0.0000016 & 0.0000012 & 0.0000009 & 0.0000007 & 0.0000005 \\
\hline
\end{tabular}

By using the simplest double-sided Bernstein - Keropian estimators it is possible to calculate the following, with under and over score, Jaroszewicz et al. [15]

$$
\Lambda_{-}=\left(\frac{A_{0}}{\sqrt{A_{1}^{2}-2 A_{0} A_{2}}}\right)^{\frac{1}{2}} ; \quad \Lambda_{+}=\left(\frac{2 A_{0}}{A_{1}+\sqrt{A_{1}^{2}-4 A_{0} A_{2}}}\right)^{\frac{1}{2}} .
$$

The results of calculation of the fundamental frequencies coefficient corresponding to Eq.(2.5) are shown in Tab.2. 
Table 2. Values of estimators of the basic frequency coefficient.

\begin{tabular}{|c|c|c|c|c|c|c|}
\hline$v$ & 0 & $1 / 9$ & $2 / 9$ & $3 / 9$ & $4 / 9$ & 0,5 \\
\hline$\gamma_{-}$ & 18.65 & 19.61 & 20.76 & 22.23 & 24.23 & 25.30 \\
\hline$\gamma_{+}$ & 18.68 & 19.64 & 20.79 & 22.26 & 24.27 & 25.33 \\
\hline
\end{tabular}

The basic frequency, as seen in Eq.(3.3), is defined by the following formula

$$
\omega_{ \pm}=\gamma_{ \pm}(v) \frac{1}{R^{2}} \sqrt{\frac{D_{0}}{\rho h_{0}}}
$$

or

$$
\omega_{ \pm}=\gamma_{ \pm}(v) \frac{h_{0}}{R^{2}} \sqrt{\frac{E}{\rho \cdot 12\left(1-v^{2}\right)}} .
$$

If we take into account that $\gamma(0)=18.7$ and $\gamma(0.5)=25.3$, it is concluded that the increase of $v$ - from 0 to 0.5 - is accompanied by an increase of the basic frequency coefficient $\gamma$ by about $35 \%$ of the disc.

In order to compare the frequency of the vibrations of a plate made of different materials it is necessary to use the formula (3.6). If, e.g., the first material is aluminum (I), and the second - tin (II) then

$$
\left(\frac{E}{\rho}\right)_{I}=2.65 ; \quad\left(\frac{\rho}{E}\right)_{I I}=7.09 ; \quad \frac{1-v_{I I}^{2}}{1-v_{I}^{2}}=\frac{0.81}{0.88}
$$

where formula (3.6) gives a result below

$$
\begin{aligned}
& \frac{(\omega)_{I}}{(\omega)_{I I}}=\frac{\gamma_{I}}{\gamma_{I I}} \sqrt{\left(\frac{E}{\rho}\right)_{I} \cdot\left(\frac{\rho}{E}\right)_{I I} \frac{1-v_{I I}^{2}}{1-v_{I}^{2}}} \approx 3.8, \\
& v_{I}=0.34 ; \quad v_{I I}=0.44 ; \quad \gamma_{I} \approx 22.2 ; \quad \gamma_{I} \approx 24.2 .
\end{aligned}
$$

\section{Calculation results and summary}

If $v$ increases, the coefficient of $\gamma(v)$ for the disc $(m=-1)$ also monotonically increases (Fig.2), so $\gamma_{\mathrm{I}}$ $/ \gamma_{\mathrm{II}}<1$. As for the diaphragm-type plate $(m>0)$, the opposite effect of $v$ is observed, and hence $v \gamma \mathrm{I} / \gamma \mathrm{II}>1$ and this means that the coefficient $v$ increases the frequency rate compared to a plate of constant thickness $\left(\omega_{I} / \omega_{I I}\right)_{\mid m=0}=4.16$. In contrast, in the disc- type plate, this ratio decreases as obtained from the solutions, and shown in Tab.3.

Table 3. Values of fundamental frequency ratio for a plate of uniform thickness, diaphragm-type plate and a disc made of duralumin (I) or tin (II).

\begin{tabular}{|c|c|c|c|}
\hline$m$ & 0 & 2 & -1 \\
\hline$\left(\omega_{I} / \omega_{I I}\right)$ & 4.16 & 4.66 & 3.83 \\
\hline
\end{tabular}


Figure 2 presents an approximation curve of dependency of the basic frequency estimators on Poisson's ratio using polynomial $\gamma=0.0704 v^{2}+0.8964 v+17.624$, Jaroszewicz and Zoryj [14].

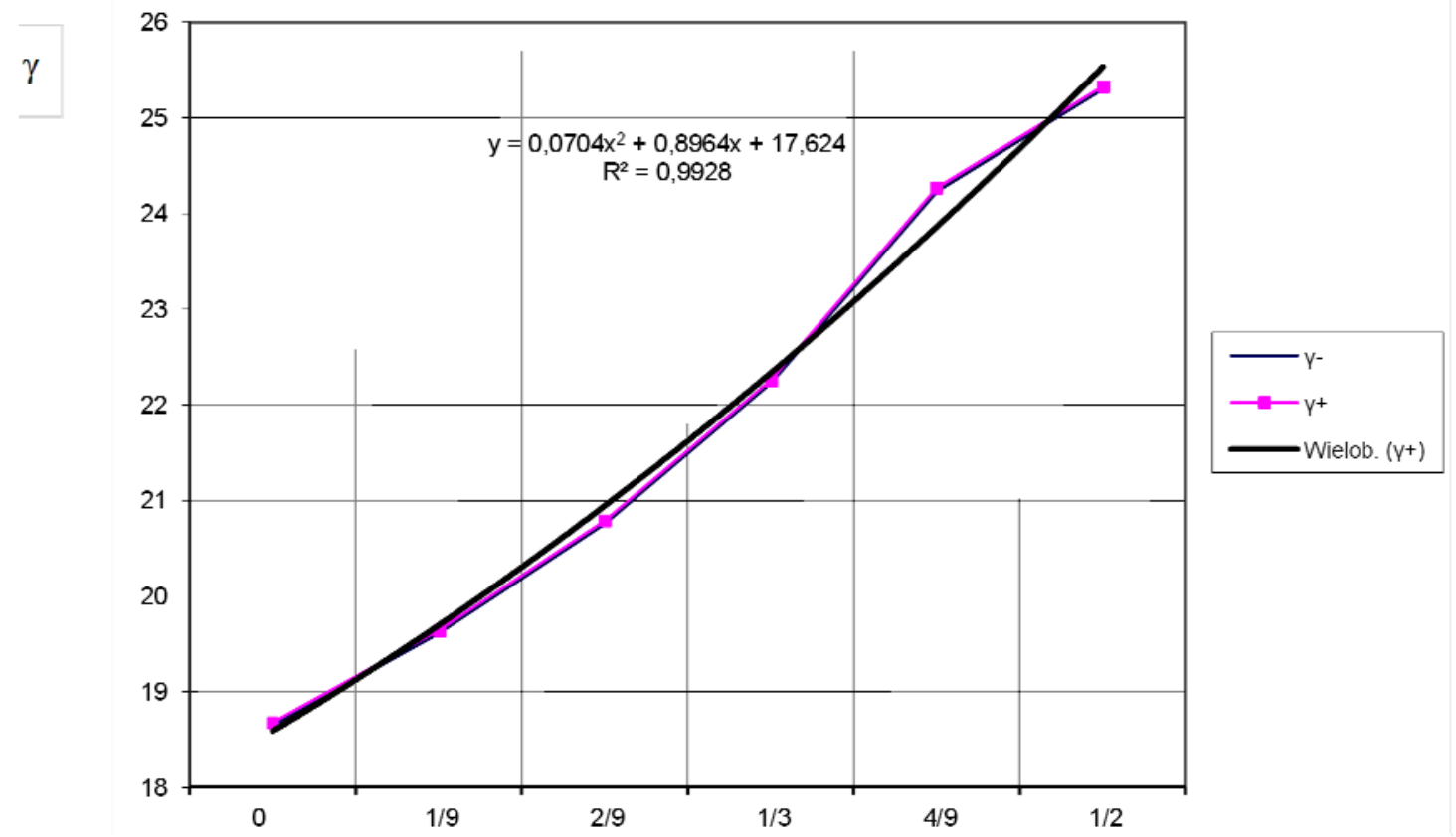

Fig.2. Dependency of estimators of basic frequency coefficients on Poisson's ratio for a disc $(m=-1)$.

This polynomial can be used in the initial calculation of the coefficient of the basic frequency of the transverse vibration of circular, disc-type plates, where $m=-1$ and $v=0 \div 0.5$. Similarly, it is possible to determine other values for the fundamental frequency $\gamma$ of other values of $m<0$. It can be noted here that the basic frequency of the disc $(m=-1)$ may be greater than the frequency of the plate with constant thickness $(m=0)$, made of the same material from $83 \%$ for $v=0$ to $247 \%$ for $v=0.5$, respectively.

\section{Conclusions}

In this paper, the Cauchy function has been employed to solve natural vibration of hyperbolic circular thin plates with a clamped edge. The limited solutions of Euler equation expanded in the power series make it possible to obtain characteristic equations of circular plates rapidly convergent to exact eigenvalues. The characteristic equations were obtained for the value of parameter $m=-1$ and different values of Poisson's ratio. The influence of the values of Poisson's ratio on the dimensionless eigenvalue was presented. The numerical results of the investigation can be used to validate the accuracy of other numerical methods as benchmark values. The calculations are made with the help of Mathematica v10.

\section{References}

[1] Leissa A.W. (1969): Vibration of Plates. - Washington.

[2] Conway H.D. (1957): An analogy between the flexural vibrations of a cone and a disc of linearly varying thickness. - Journal of Applied Mathematics and Mechanics, vol.37, No.9, pp.406-407.

[3] Conway H.D. (1958): Some special solutions for the flexural vibrations of discs of varying thickness. - IngenieurArchiv, vol.26, No.6, pp.408-410. 
[4] Jain R.K., Prasad C. and Soni S.R. (1972): Axisymmetric vibrations of circular plates of linearly varying thickness. - ZAMP, vol.23, pp.941-947.

[5] Wang J. (1997): General power series solution of the vibration of classical circular plates with variable thickness. - Journal of Sound and Vibration, vol.202, pp.593-599.

[6] Wu T.Y. and Liu G.R. (2001): Free vibration analysis of circular plates with variable thickness by the generalized differential quadrate rule. - International Journal of Solids and Structures, vol.38, pp.7967-7980.

[7] Wu T.Y. and Liu G.R. (2002): Free vibration analysis of circular plates using generalized differential quadrature rule. - Computer Methods in Applied Mechanics and Engineering, vol.191, pp.5365-5380.

[8] Jaroszewicz J. and Zoryj L. (2006): The method of partial discretization in free vibration problems of circular plates with variable distribution of parameters. - International Applied Mechanics, vol.42, pp.364-373.

[9] Zhou Z.H., Wong K.W., Xu X.S. and Leung A.Y.T. (2011): Natural vibration of circular and annular plates by Hamiltonian Approach. - Journal of Sound and Vibration, vol.330, No.5, pp.1005-1017.

[10] Duan G., Wang X. and Jin Ch. (2014): Free vibration analysis of circular thin plates with stepped thickness by the DSC element method. - Thin-Walled Structures, vol.85, pp.25-33.

[11] Żur K.K. (2015): Green's function in frequency analysis of circular thin plates of variable thickness. - Journal of Theoretical and Applied Mechanics, vol.53, No.4, pp.873-884.

[12] Żur K.K. (2016a): Green's function approach to frequency analysis of thin circular plates. - Bulletin of the Polish Academy of Sciences - Technical Sciences, vol.64, No.1, pp.181-188.

[13] Żur K.K. (2016b): Green's function in frequency analysis of thin annular plates of nonlinear thickness variation.Applied Mathematical Modelling, vol.40, pp.5-6, pp.3601-3619.

[14] Jaroszewicz J. and Zoryj L. (2005): Method of free aximmetric vibration analysis of circular plates via method of influece Cauchy function - Rozprawy Naukowe Politechniki Białostockiej Nr 124, Białystok, pp.120.

[15] Jaroszewicz J., Misiukiewicz M. and Puchalski W. (2008): Limitations in application of basic frequency simplest lower estimators in investigation of natural vibrations circular plates with variable thickness and clamped edges. Journal of Theoretical and Applied Mechanics, vol.46, No.1, pp.109-121.

Received: July 15, 2016

Revised: March 13, 2017 